\title{
Propuesta de validación por jueces expertos de una intervención cognitivo-conductual para cuidadores primarios de pacientes con cáncer
}

\author{
Validation proposal by expert judges of a cognitive-behavioural intervention for \\ primary caregivers of cancer patients \\ Karla Salinas Ríos ${ }^{a}$, Andrómeda I. Valencia Ortiz ${ }^{b}$, Rubén García Cruz ${ }^{c}$, Flor M. \\ E. Gil Bernal ${ }^{d}$
}

\begin{abstract}
:
The incidence and prevalence of chronic-degenerative diseases (which demand constant care) such as cancer, has increased in recent years, and therefore, there has also been an increase in the number of informal caregivers When talking about informal care, in many cases, it is only one person (usually a relative) who is responsible for meeting the needs of the patient and who is called primary caregiver. However, the demands of such work can have negative consequences at a physical, psychosocial and cognitive level, dimensions of Quality of Life Related to Health (HRQoL). It is worth mentioning that the experience, good or bad, of each caregiver is different in each case, since there are several factors that influence their quality of life throughout the care process, including the coping strategies they have. Objective: To develop and validate by expert judges a cognitive-behavioural intervention to increase the level of quality of life and develop adaptive coping strategies in primary caregivers of cancer patients. Method: through a non-probabilistic sample for convenience with a probable sample of 15 expert judges. The type of study is descriptive with a non experimental, transversal design. The instrument to be used will be a questionnaire of expert judges. Expected results: In general, it is expected that the intervention will be adequate for this type of population, that it is designed to meet the stated objectives and that it meets the determined ethical criteria.
\end{abstract}

Keywords:

Cancer, primary caregiver, quality of life, coping strategies, cognitive-behavioural intervention, validation

\section{Resumen:}

La incidencia y prevalencia de enfermedades crónico-degenerativas (que demandan cuidado constante) como lo es el cáncer, se ha incrementado en los últimos años, y por ende, también ha habido un aumento en el número de cuidadores informales. Cuando se habla del cuidado informal, en muchas ocasiones, es sólo una persona (normalmente un familiar) la que se encarga de atender las necesidades del paciente y al que se le denomina cuidador primario. Sin embargo, las demandas de dicha labor pueden traer consecuencias negativas a nivel físico, psicosocial y cognitivo, dimensiones de la Calidad de Vida Relacionadas a la Salud (CVRS). Cabe mencionar que la experiencia, buena o mala, de cada cuidador es diferente en cada caso, ya que son varios factores los que influyen en su calidad de vida a lo largo del proceso de cuidado, entre ellos las estrategias de afrontamiento con las que cuenta. Objetivo: Desarrollar y validar por jueces expertos una intervención cognitivo-conductual para incrementar el nivel de la calidad de vida y desarrollar estrategias de afrontamiento adaptativas en cuidadores primarios de pacientes con cáncer. Método: a través de una muestra no probabilística por conveniencia con una muestra probable de 15 jueces expertos. El tipo de estudio es descriptivo con un diseño no experimental, transversal. El instrumento que se utilizará será un cuestionario de jueces expertos. Resultados esperados: De manera general se espera que la intervención sea adecuada para este tipo de población, que esté diseñada para cumplir con los objetivos planteados y que cumpla con los criterios éticos determinados.

\section{Palabras Clave:}

Cáncer, cuidador primario, calidad de vida, estrategias de afrontamiento, intervención cognitivo-conductual, validación

\footnotetext{
Autor de Correspondencia, Universidad Autónoma del Estado de Hidalgo, Instituto de Ciencias de la Salud, Email: salinaskarla93@gmail.com

${ }^{b}$ Universidad Autónoma del Estado de Hidalgo, Instituto de Ciencias de la Salud, https://orcid.org/0000-0001-9664-1993, Email: andromeda_valencia@uaeh.edu.mx

${ }^{\mathrm{c}}$ Universidad Autónoma del Estado de Hidalgo, Instituto de Ciencias de la Salud, Email: rubengarciacruz2014@gmail.com

d Universidad Autónoma del Estado de Hidalgo, Instituto de Ciencias de la Salud, https://orcid.org/0000-0001-7756-3125, Email: florerari@yahoo.com
} 


\section{Introducción}

En los últimos años el número de personas con cáncer ha ido creciendo a nivel mundial, esto debido a las tasas de incidencia y de supervivencia, convirtiéndose así, en un problema grave de salud. Además, debido a los avances científicos, el cáncer se ha transformado en una enfermedad crónico-degenerativa, a comparación de años atrás donde era visto como una enfermedad fatal. Este cambio en su condición, ha requerido que al mismo tiempo aumente el número de cuidadores informales. 1,2

El cuidador informal es aquel que brinda cuidado y atención a familiares o personas con las que tiene un lazo emocional-afectivo, dicha labor es brindada a personas que tienen algún grado de discapacidad o dependencia. Dentro de sus actividades están el proveer apoyo emocional, dar medicamentos y ayudar en quehaceres domésticos, siendo el cuidador primario quien tiene mayores responsabilidades. 3,4

Cuando se habla de las consecuencias que ocasiona el cáncer, generalmente se centra en ver cómo repercuten en el enfermo, sin embargo, no se deben minimizar las repercusiones que hay en las personas que rodean al paciente y en particular en lo que le ocurre al cuidador primario. En este sentido, diversos estudios han sugerido que la condición de cuidar a un paciente oncológico es en sí una situación estresante que deriva en consecuencias físicas, psicosociales y cognitivas, dimensiones de la Calidad de Vida Relacionada con la Salud (CVRS). 5-7

Cabe mencionar que la experiencia, buena o mala, de cada cuidador es diferente en cada caso, ya que son varios factores los que influyen en su calidad de vida a lo largo del proceso de cuidado, entre ellos las estrategias de afrontamiento con las que cuenta. Es decir, no todos los cuidadores afrontan de la misma manera la situación de cuidar a un enfermo, hay algunos que lo hacen de mejor manera que otros. Esto es relevante ya que un afrontamiento adecuado está relacionado con bienestar psicológico y emocional, un buen funcionamiento social y buen estado de salud física. 6,8-10

En este sentido, la presente investigación se propone diseñar un programa de intervención que ayude a mejorar la calidad de vida y a desarrollar estrategias de afrontamiento adaptativas en cuidadores primarios de pacientes con cáncer. Destacando que dicha intervención será diseñada con base en una revisión teórica previa para que posteriormente se valide por jueces expertos en el área de la psicología, la terapia cognitivo-conductual y de los cuidadores primarios de pacientes oncológicos.

\section{Marco teórico}

Como se mencionó anteriormente, la incidencia y prevalencia de enfermedades crónico-degenerativas (que demandan cuidado constante) como lo es el cáncer, se ha incrementado en los últimos años, y por ende, también ha habido un aumento en el número de cuidadores informales. Cuando se habla del cuidado informal, en muchas ocasiones, es sólo una persona (normalmente un familiar) la que se encarga de atender las necesidades del paciente y al que se le denomina cuidador primario. 2,6,11

Por muchos años en la relación enfermo-cuidador, los profesionales de la salud sólo prestaron atención a la persona que padecía la enfermedad. Especialmente en el campo oncológico, el bienestar del cuidador no era tomado en cuenta por el simple hecho de que éste último no era el que estaba diagnosticado con cáncer. Sin embargo, en los últimos años diversos profesionales asociados al cuidado del enfermo oncológico, le han dado mayor importancia al estudio de esta figura. 12,13 La reciente focalización en el cuidador primario se debe al papel esencial que funge dentro del campo de la salud, especialmente dentro del Sistema de Cuidado Domiciliario ya que es este quien continúa con la atención médica que el Sistema Formal no puede, adquiere la carga principal de los cuidados del enfermo, y funge como una figura intermedia entre equipo médico-paciente y entre el paciente-entorno familiar o social. Además, diversos estudios han sugerido que la condición de cuidar a un paciente oncológico es en sí una situación estresante que deriva en consecuencias físicas, psicosociales y cognitivas, dimensiones de la Calidad de Vida Relacionada con la Salud (CVRS). $6,7,14$

Debido a las repercusiones derivadas de su tarea, es necesario no sólo conocer más a acerca de ellas (pese a la gran cantidad de investigaciones que ya existen documentadas), si no se plantea la necesidad de establecer estrategias de prevención e intervención que permitan mejorar su calidad de vida. 15,16,17

Reforzando lo anterior, en los últimos años, el desarrollo de intervenciones psicosociales para abordar distintas necesidades en cuidadores primarios de pacientes con cáncer ha ido en aumento, lográndose un gran número de éstas. Con base en los datos de diversas revisiones 
sistemáticas, a continuación, se mencionan las características principales y comunes de algunos programas aplicados a la población ya antes mencionada. Esto, con el objetivo de tener un panorama general de lo que se ha hecho en este campo. 17 Dichas intervenciones:

- Pertenecen o tienen componentes de las siguientes categorías: psicoeduactivas, solución de problemas o desarrollo de habilidades, terapia de apoyo, terapia familiar, terapia cognitivo conductual, terapia interpersonal, intervenciones complementarias o de medicina alternativa y terapia existencial. Siendo las psicoeducativas las que más se aplican junto con las integradoras. Las últimas, además, son las más factibles y las que ofrecen mayores resultados. 16,17

- Tienen tres principales modalidades: cara a cara, por teléfono o la combinación de ambas. Siendo las primeras las más usuales. 16,17

- También pueden ser individuales, grupales o de díadas (paciente-cuidador). 16-18

- Tienen un número de sesiones que van de 1 a 16 . 16,17

- Son aplicadas por enfermeras, trabajadores sociales, psicólogos, oncólogos o la combinación de estos profesionales. Siendo el área de enfermería la que más destaca. 16,18

- De manera general, tienen resultados positivos y significativos, pero el tamaño del efecto va de pequeño a mediano. 16-18

- Dirigidas específicamente a modificar la calidad de vida y las estrategias de afrontamiento, arrojaron los siguientes resultados: el efecto para las primera variable va de pequeño a moderado con la presencia de algunos cambios. Por el contrario, para la segunda variable, el efecto es positivo y significativo. 16,18

Cabe destacar, que ninguno de los estudios incluidos en las revisiones fue realizado en México. La mayoría de las investigaciones se llevaron a cabo en Estados Unidos. 16-18

Por otro lado, pese al número considerable de intervenciones que se han desarrollado en cuidadores primarios de pacientes con cáncer, es importante mencionar, que la investigación en este campo todavía es considerada relativamente nueva, limitada, particular (por los desafíos exclusivos del cáncer a los que se enfrentan) y con poco saber acerca de la efectividad de las intervenciones. 16-19

Tomando en cuenta lo anterior, se sugiere que:

- Se preste atención a estudios que no solo sean ensayos clínicos aletorizados. 17

- Se diseñen intervenciones que incorporen tecnología, que sean eficaces (o bien que muestran alguna mejoría), rentables, accesibles y que puedan llevarse a la práctica. Este último punto es de gran relevancia debido a que se ha demostrado que algunos programas no tienen esta capacidad. 19,20

- Se pueda incorporar al cuidador a contextos de salud formales con dichas intervenciones, ya que los sistemas de salud no cuentan con los elementos necesarios para poder hacerlo. 19

- Además de la significancia estadística se considere la significancia clínica, ya que esta última puede estar relacionada con el tamaño del efecto (aunque este sea pequeño). 16

Además del poco conocimiento que se tiene acerca de la efectividad de las intervenciones en cuidadores primarios de pacientes con cáncer, se presenta otro problema de igual magnitud: la falta de intervenciones validadas en este campo.

Según Martín, en general, las intervenciones psicológicas deben estar determinadas por características propias 0 específicas de cada enfermedad, como sus limitaciones o síntomas y el cáncer no es la excepción. 21

Con base en los dos puntos anteriores, surge la necesidad de diseñar intervenciones psicológicas específicas para el campo oncológico, mismas que se validen por jueces expertos. Así, se podrá determinar si dichas intervenciones son adecuadas para ese tipo de población (técnicas, materiales, procedimiento), si están diseñadas para cumplir con los objetivos planteados y si cumplen con los criterios éticos requeridos.

De esta manera, el objetivo de la presente investigación es diseñar y validar por jueces expertos una intervención cognitivo-conductual para incrementar el nivel de la calidad de vida y desarrollar estrategias de afrontamiento adaptativas en cuidadores primarios de pacientes con cáncer.

\section{Pregunta de investigación}

¿Las características de una propuesta de intervención cognitivo-conductual para incrementar el nivel de la calidad de vida y desarrollar estrategias de afrontamiento adaptativas en cuidadores primarios de pacientes con cáncer cumplen con los criterios metodológicos para su validación por jueces expertos?

\section{Objetivos}

\section{Objetivo general}

Desarrollar y validar por jueces expertos una intervención cognitivo-conductual para incrementar el nivel de la calidad de vida y desarrollar estrategias de 
afrontamiento adaptativas en cuidadores primarios de pacientes con cáncer que cumpla con los criterios éticos y los componentes necesarios para ser una intervención basada en evidencia.

\section{Objetivos específicos}

- Diseñar una propuesta de intervención cognitivoconductual para incrementar el nivel de la calidad de vida y desarrollar estrategias de afrontamiento adaptativas en cuidadores primarios de pacientes con cáncer.

- Desarrollar materiales de uso clínico para una intervención cognitivo-conductual para incrementar el nivel de la calidad de vida y desarrollar estrategias de afrontamiento adaptativas en cuidadores primarios de pacientes con cáncer.

- Validar por jueces expertos la propuesta de intervención cognitivo-conductual para incrementar el nivel de la calidad de vida y desarrollar estrategias de afrontamiento adaptativas en cuidadores primarios de pacientes con cáncer, que cumpla con los criterios éticos y los componentes necesarios para ser una intervención basada en evidencia.

\section{Hipótesis}

Hi: Las características de una propuesta de intervención cognitivo-conductual para incrementar el nivel de la calidad de vida y desarrollar estrategias de afrontamiento adaptativas en cuidadores primarios de pacientes con cáncer cumplen con los criterios metodológicos para su validación por jueces expertos.

Ho: Las características de una propuesta de intervención cognitivo-conductual para incrementar el nivel de la calidad de vida y desarrollar estrategias de afrontamiento adaptativas en cuidadores primarios de pacientes con cáncer no cumplen con los criterios metodológicos para su validación por jueces expertos.

\section{Método}

\section{Participantes}

Los participantes están integrados por expertos en el área de psicología de la salud, terapia cognitivoconductual y cuidadores primarios de pacientes con cáncer.

\section{Criterios de inclusión}

- Tener grado académico de licenciatura, maestría o doctorado en el área de psicología.

- Tener experiencia en el área de intervención cognitivo-conductual.
- Que acepte la participación como jurado en la aprobación de la propuesta de intervención.

\section{Criterios de eliminación}

- Que no responda el cuestionario proporcionado.

- Que no entregue el resultado de su evaluación en el tiempo requerido.

\section{Tipo de estudio y diseño}

Descriptivo, ya que se llevarán a cabo mediciones en un grupo de personas en una o más variables y sólo se describirán los efectos observados. 22

\section{Tipo de diseño}

No experimental, transversal, ya que las valoraciones que emitan los jueces expertos serán en un solo momento de tiempo. 23

\section{Tipo de muestreo}

No probabilístico, por conveniencia, con una muestra probable de 15 jueces expertos (5 en el campo de Psicología de la Salud, 5 en el campo de la terapia cognitivo-conductual y 5 en el campo de cuidadores primarios de pacientes con cáncer). Número aceptable de acuerdo a Hyrkas, Appelqvist-Schmidlechner y Oksa, quienes plantean que la validez de contenido posee una estimación confiable teniendo como mínimo 10 jueces expertos. 24

\section{Instrumentos de medición}

Cuestionario de jueces expertos para la validación de la intervención cognitivo-conductual para incrementar el nivel de la calidad de vida y desarrollar estrategias de afrontamiento adaptativas en cuidadores primarios de pacientes con cáncer.

Este instrumento constituye el único indicador de la validez de contenido y fue diseñado con base en el trabajo de Wolf. Su aplicación es digital y consta de 13 preguntas, mismas que evalúan características relacionadas a la intervención como: metas, procedimientos y resultados. 25

\section{Procedimiento}

Como primer paso se realizará una revisión teórica de las principales características y técnicas cognitivoconductuales que se han utilizado en el campo.

Posteriormente, se seleccionarán aquellas que sean adecuadas para la implementación de la intervención y que servirán para diseñar la propuesta.

A continuación, se procederá a seleccionar la muestra, teniendo en cuenta los criterios de inclusión y exclusión. También se elaborará una presentación, cuyo objetivo será exponer el contenido de la intervención diseñada. 
Seguido de esto, se extenderá la invitación a los jueces expertos, y se les enviará la presentación y el cuestionario digital para que lo contesten (vía correo electrónico).

Finalmente, se recolectará la información, se analizará y se realizarán los ajustes o correcciones necesarias de acuerdo al criterio de los jueces expertos.

\section{Análisis de datos}

Análisis estadístico descriptivo utilizando el Paquete de Datos Estadístico para las Ciencias Sociales (SPSS) versión 21.

\section{Resultados esperados}

De manera general se espera que la intervención sea adecuada para este tipo de población, que esté diseñada para cumplir con los objetivos planteados y que cumpla con los criterios éticos determinados.

Finalmente, con base en la revisión de la literatura que hasta el momento se ha realizado, a continuación, se hace una propuesta preliminar de la intervención, misma que se irá complementando, modificando y mejorando antes y después de su presentación ante los jueces expertos.

Como se ha mencionado anteriormente, dicha propuesta es de tipo cognitivo-conductual y consta de 12 sesiones cada una con una duración de 2 hrs. Los temas centrales que se abordarán serán el autocuidado, el entrenamiento en habilidades sociales y la solución de problemas, distribuidos en las 12 sesiones, como se observa en la Tabla 1.

Tabla 1

Tabla descriptiva de los contenidos propuestos por sesión

\begin{tabular}{|l|l|l|}
\hline Sesión & Tema & Objetivo \\
\hline 1 & $\begin{array}{l}\text { Entrevista de } \\
\text { primer contacto }\end{array}$ & $\begin{array}{l}\text { Realizar una entrevista para la } \\
\text { obtención de datos relevantes. }\end{array}$ \\
\cline { 2 - 3 } & Pre-evaluación & $\begin{array}{l}\text { Batería: Formato de } \\
\text { consentimiento informado } \\
\text { Hoja de datos } \\
\text { sociodemográficos } \\
\text { Cuestionario WHOQOL-BREF } \\
\text { Cuestionario de Afrontamiento } \\
\text { del Estrés }\end{array}$ \\
\hline
\end{tabular}

\begin{tabular}{|c|c|c|}
\hline \multirow[t]{2}{*}{2} & Presentación & $\begin{array}{l}\text { Presentar y explicar el } \\
\text { contenido de la intervención a } \\
\text { los participantes. }\end{array}$ \\
\hline & $\begin{array}{l}\text { Cáncer e } \\
\text { introducción al } \\
\text { cuidado }\end{array}$ & $\begin{array}{l}\text { Realizar psicoeducación de } \\
\text { diversos temas como: cáncer, } \\
\text { estrategias para el cuidado y } \\
\text { consecuencias del cuidado. }\end{array}$ \\
\hline \multirow[t]{2}{*}{3} & Retroalimentación & $\begin{array}{l}\text { Retroalimentar los aspectos o } \\
\text { temas revisados en la sesión } \\
\text { pasada }\end{array}$ \\
\hline & Autocuidado & $\begin{array}{l}\text { Realizar psicoeducación } \\
\text { acerca de la importancia del } \\
\text { autocuidado, así como de las } \\
\text { estrategias o actividades para } \\
\text { el mismo. }\end{array}$ \\
\hline \multirow[t]{2}{*}{4} & Retroalimentación & $\begin{array}{l}\text { Retroalimentar los aspectos o } \\
\text { temas revisados en la sesión } \\
\text { pasada. }\end{array}$ \\
\hline & Autocuidado & $\begin{array}{l}\text { Modelar y entrenar al cuidador } \\
\text { en distintas técnicas } \\
\text { psicológicas como la } \\
\text { respiración, mismas que le } \\
\text { servirán para su autocuidado. }\end{array}$ \\
\hline \multirow[t]{2}{*}{5} & Retroalimentación & $\begin{array}{l}\text { Retroalimentar los aspectos o } \\
\text { temas revisados en la sesión } \\
\text { pasada. }\end{array}$ \\
\hline & Autocuidado & $\begin{array}{l}\text { Modelar y entrenar al cuidador } \\
\text { en actividades } \\
\text { complementarias para su } \\
\text { cuidado. }\end{array}$ \\
\hline \multirow[t]{2}{*}{6} & Retroalimentación & $\begin{array}{l}\text { Retroalimentar los aspectos o } \\
\text { temas revisados en la sesión } \\
\text { pasada. }\end{array}$ \\
\hline & $\begin{array}{l}\text { Entrenamiento en } \\
\text { habilidades } \\
\text { sociales }\end{array}$ & $\begin{array}{l}\text { Explicar qué es la asertividad } \\
\text { y brindar estrategias para } \\
\text { llevarla a cabo. }\end{array}$ \\
\hline \multirow[t]{2}{*}{7} & Retroalimentación & $\begin{array}{l}\text { Retroalimentar los aspectos o } \\
\text { temas revisados en la sesión } \\
\text { pasada. }\end{array}$ \\
\hline & $\begin{array}{l}\text { Entrenamiento en } \\
\text { habilidades } \\
\text { sociales }\end{array}$ & $\begin{array}{l}\text { Explicar qué es la negociación } \\
\text { y brindar estrategias para } \\
\text { llevarla a cabo. }\end{array}$ \\
\hline
\end{tabular}




\begin{tabular}{|c|c|c|}
\hline \multirow[t]{2}{*}{8} & Retroalimentación & $\begin{array}{l}\text { Retroalimentar los aspectos o } \\
\text { temas revisados en la sesión } \\
\text { pasada. }\end{array}$ \\
\hline & $\begin{array}{l}\text { Entrenamiento en } \\
\text { habilidades } \\
\text { sociales }\end{array}$ & $\begin{array}{l}\text { Explicar qué es la toma de } \\
\text { decisiones y brindar } \\
\text { estrategias para llevarla a } \\
\text { cabo. }\end{array}$ \\
\hline \multirow[t]{2}{*}{9} & Retroalimentación & $\begin{array}{l}\text { Retroalimentar los aspectos o } \\
\text { temas revisados en la sesión } \\
\text { pasada. }\end{array}$ \\
\hline & $\begin{array}{l}\text { Solución de } \\
\text { problemas }\end{array}$ & $\begin{array}{l}\text { Brindar estrategias para la } \\
\text { solución de problemas. }\end{array}$ \\
\hline \multirow[t]{2}{*}{10} & Retroalimentación & $\begin{array}{l}\text { Retroalimentar los aspectos o } \\
\text { temas revisados en la sesión } \\
\text { pasada. }\end{array}$ \\
\hline & $\begin{array}{l}\text { Solución de } \\
\text { problemas }\end{array}$ & $\begin{array}{l}\text { Brindar estrategias para la } \\
\text { solución de problemas. }\end{array}$ \\
\hline \multirow[t]{2}{*}{11} & Retroalimentación & $\begin{array}{l}\text { Retroalimentar los aspectos o } \\
\text { temas revisados en la sesión } \\
\text { pasada. }\end{array}$ \\
\hline & $\begin{array}{l}\text { Entrenamiento en } \\
\text { habilidades } \\
\text { sociales }\end{array}$ & $\begin{array}{l}\text { Explicar qué es la toma de } \\
\text { decisiones y brindar } \\
\text { estrategias para llevarla a } \\
\text { cabo. }\end{array}$ \\
\hline \multirow[t]{2}{*}{12} & Post-evaluación & $\begin{array}{l}\text { Batería: Hoja de datos } \\
\text { sociodemográficos } \\
\text { Cuestionario WHOQOL-BREF } \\
\text { Cuestionario de Afrontamiento } \\
\text { del Estrés }\end{array}$ \\
\hline & Conclusión & $\begin{array}{l}\text { Retroalimentar y finalizar el } \\
\text { programa de intervención }\end{array}$ \\
\hline
\end{tabular}

\section{Comentarios finales}

Las estrategias seleccionadas han mostrado su efectividad en investigaciones previas, por lo que se espera que de acuerdo a su expertis los jueces identifiquen la pertinencia de las mismas.

\section{Referencias}

[1] International Agency for Research on Cancer. GLOBOCAN 2012: Estimated cancer incidence, mortality and prevalence worldwide in 2012; 2016. Recuperado de http://globocan.iarc.fr/Default.aspx

[2] Masa'Deh R, Collier J, Hall C. Parental strees when caring for a child with cancer in Jordan: A cross-sectional survey. Health and Quality of Life Outcomes 2012; 10(88): 1-7.
[3] Gómez-Martinho MR. Cuidado formal e informal de personas mayores dependientes; $2016 . \quad$ Recuperado de https://repositorio.comillas.edu/xmlui/bitstream/handle/11531/13437/T FM000507.pdf?sequence $=1$

[4] American Society of Clinical Oncology. Being a Caregiver; 2015. Recuperado de http://www.cancer.net/coping-with-cancer/caringloved-one/being-caregiver

[5] Barrón BS, Alvarado S. Desgaste físico y emocional del cuidador primario en cáncer. Cancerología 2009; 4: 39-46. Recuperado de http://www.antesdepartir.org.mx/lecturas/Desgaste-del-cuidador.pdf

[6] Mesa-Gresa P, Ramos-Campos M, Redolat R. Cuidado de pacientes oncológicos: una revisión sobre el impacto de la situación de estrés crónico y su relación con la personalidad del cuidador y otras variables moduladoras. Psicooncología 2017; 14(1): 93-106. doi: 10.5209/PSIC.55814

[7] Torres RP. Del impacto en la calidad de vida como consecuencia de la enfermedad, los instrumentos de medición y otras reflexiones. Revista CES Salud Pública 2012; 3(1): 108-115. Recuperado de https://dialnet.unirioja.es/descarga/articulo/3977659.pdf

[8] Ruíz AE, Nava MG. Cuidadores: responsabilidades-obligaciones. Enfermería Neurológica 2012: 11(3): 163-169. Recuperado de http://www.medigraphic.com/pdfs/enfneu/ene-2012/ene123i.pdf

[9] Torres IA, Beltrán FJ, Martínez G, Saldívar AH, Quesada J, Cruz LD. Cuidar a un enfermo ¿pesa? Revista de Divulgación Científica y Tecnológica de la Universidad Veracruzana 2006; 19(2):, 47-50. Recuperado de http://www.uv.mx/cienciahombre/revistae/vol19num2/articulos/cuidar/

[10] Sánchez CL. Estilos de personalidad, afrontamiento y satisfacción en profesionales sanitarios en relación con la salud (Tesis de doctorado); 2016. Recuperado de https://eprints.ucm.es/37677/1/T37218.pdf

[11] Astudillo W, Mendinueta C. Necesidades de los cuidadores del paciente crónico. En Guía de Recursos Sanitarios y Sociales en la Fase Final de la Vida en Gipuzkoa 2003 (pp. 235-255). España: Sociedad Vasca de Cuidados Paliativos.

[12] Northouse LL, Katapodi MC, Schafenacker AM, Weiss D. The impact of caregiving on the psychological well-being of family caregivers and cancer patients. Seminars in Oncology Nursing 2012; 28(4): 236-245. doi: 10.1016/j.sonen.2012.09.006

[13] Caqueo-Urízar A, Segovia-Lagos P, Urrutia-Urrutia U, Miranda C, Navarro E. Impacto de la relación de ayuda de cuidadores primarios en la calidad de vida de pacientes con cáncer avanzado. Psicooncología 2013; 10(1): 95-108. doi: 10.5209/rev_PSIC.2013.v10.41950

[14] Romero E. La familia del paciente paliativo. "Hablan las cuidadoras"; 2008. Recuperado el 22 de junio de 2016 de http://www.humanizar.es/uploads/media/08_atencion_a_la_familia_de 1_enfermo_XIII_Jorn.pdf

[15] Mendonca MD, Alves L, Bugalho P. From subjective cognitive complaints to dementia: Who is at Risk? A systematic review. American Journal of Alzheimers Disease \& Other Dementias 2015; 31(2): 105-14. doi: 10.1177/1533317515592331

[16] Northouse LL, Katapodi MC, Schafenacker AM, Weiss D. The impact of caregiving on the psychological well-being of family caregivers and cancer patients. Seminars in Oncology Nursing 2012; 28(4): 236-245. doi: 10.1016/j.sonen.2012.09.006

[17] Applebaum AJ, Breitbart W. Care for the cancer caregiver: a systematic review. Palliat Support Care 2013;11(03): 231- 252.

[19] Kent EE, Rowland JH, Northouse L, et al. Caring for caregivers and patients: research and clinical priorities for informal cancer caregiving. Cancer 2016; 122(13): 1987- 1995

[20] Ugalde A, Gaskin CJ, Rankin NM, et al. A systematic review of cancer caregiver interventions: Appraising the potential for 
Publicación semestral, Educación y Salud Boletín Científico Instituto de Ciencias de la Salud Universidad Autónoma del Estado de Hidalgo, Vol. 8 No. 15 (2019) 144-150

implementation of evidence into practice. Psycho- Oncology. 2019;28:687-701. https://doi.org/10.1002/pon.5018

[21] Martín M. La psicología en la atención a las personas con enfermedades crónicas: experiencias y retos. Integración Académica en Psicología 2016; 4(11): 2-18.

[22] García B, Márquez L, Ávila JL. Planeación y desarrollo del proyecto de investigación 2009 (pp.1-48). Manual de métodos de investigación para las ciencias sociales. México, D.F.: Manual Moderno.

[23] Hernández R, Fernández-Collado C, Baptista P. Metodología de la investigación 2008. México: Mc Graw-Hill Interamericana.

[24] Hyrkas K, Appelqvist-Schmidlechner K, Oksa L. Validating an instrument for clinical supervision using an expert panel. International Journal of nursing studies 2003; 40(6): 619 -625.

[25] Wolf MM. Social validity: the case for subjective measurement or how applied behavior analysis is finding its heart. Journal of applied behavior analysis 1978; 11(2): 203-2014. 